\title{
Multiservice QoS-Enabled MAC for Optical Burst Switching
}

\author{
Joan Triay, Georgios S. Zervas, Cristina Cervelló-Pastor, and Dimitra Simeonidou
}

\begin{abstract}
The emergence of a broad range of network-driven applications (e.g., multimedia, online gaming) brings in the need for a network environment able to provide multiservice capabilities with diverse quality-of-service (QoS) guarantees. In this paper, a medium access control protocol is proposed to support multiple services and QoS levels in optical burst-switched mesh networks without wavelength conversion. The protocol provides two different access mechanisms, queue-arbitrated and prearbitrated for connectionless and connection-oriented burst transport, respectively. It has been evaluated through extensive simulations and its simplistic form makes it very promising for implementation and deployment. Results indicate that the protocol can clearly provide a relative quality differentiation for connectionless traffic and guarantee null (or negligible, and thus acceptable) burst loss probability for a wide range of network (or offered) load while ensuring low access delay for the higher-priority traffic. Furthermore, in the multiservice scenario mixing connectionless and connection-oriented burst transmissions, three different prearbitrated slot scheduling algorithms are evaluated, each one providing a different performance in terms of connection blocking probability. The overall results demonstrate the suitability of this architecture for future integrated multiservice optical networks.
\end{abstract}

Index Terms-Optical fiber communication; Medium access control; Optical burst switching.

\section{INTRODUCTION}

$\mathbf{T}$ riple-play services (i.e., data, voice, and video) and the new deployment of web-based multimedia applications have increased the amount of bursty traffic on the Internet. Such services may benefit from

Manuscript received December 17, 2009; revised April 29, 2010; accepted May 28, 2010; published July 14, 2010 (Doc. ID 119755).

Joan Triay (e-mail: joan.triay@entel.upc.edu) and Cristina Cervelló-Pastor are with the Department of Telematics Engineering at Universitat Politècnica de Catalunya (UPC), Esteve Terradas 7, 08860, Castelldefels, Spain.

Georgios S. Zervas and Dimitra Simeonidou are with the School of Computer Science and Electronic Engineering at University of Essex, Wivenhoe Park, CO4 3SQ, Colchester, United Kingdom.

Digital Object Identifier 10.1364/JOCN.2.000530 the utilization of packet/burst-switched networks. However, certain applications such as IPTV, together with grid and cloud computing (e.g., PC virtualization, etc.) can benefit from time division multiplexing (TDM) connection-oriented transport networks. The traffic diversity created by such applications and the rapid advance of optical technologies has driven the development of new optical network architectures able to provide flexible and dynamic resource allocation [1]. In this sense, a desirable requirement is to provide and guarantee a great variety of services over the same optical network infrastructure.

A promising technological option for the future optical Internet is optical burst switching (OBS) [2]. OBS can satisfy the future bandwidth requirements avoiding the inefficient resource utilization of optical circuit switching (OCS) and the requirements of optical packet switching (OPS) in terms of optical buffers, fast processing, and implementation complexity. Nevertheless, optical burst-switched networks without wavelength conversion may exhibit a high burst loss rate in the core of the network, especially if no other means of contention resolution are present. In view of this, we are interested in pursuing new contention resolution (or avoiding) methods to keep losses to a reasonably low level.

Medium access control (MAC) protocols can efficiently manage the huge optical bandwidth by providing contention avoidance schemes to further improve the packet/burst delivery on the network. This characteristic becomes of special interest for all-optical networks with limited contention resolution. However, its use in OBS networks has not been intensively analyzed, and most current MAC solutions have focused on metro-ring architectures, leaving out of their scope other common topologies, i.e., mesh. In this sense, in [3] an adaptation of the IEEE 802.6 Distributed Queue Dual Bus (DQDB) [4] for OBS networks (DAOBS) was introduced. DQDB defines a queuearbitrated access to the channel guaranteeing zero losses for transmitted frames. The original protocol was enhanced by adapting it over mesh network topologies and integrating it as a wavelength-aware MAC for wavelength continuity constraint slotted OBS networks. In DAOBS, a set of counters keep 
track of the requests for free slots and their availability using request and burst control packets. With this information, bursts are only transmitted when there are free resources; hence overlapped burst losses are avoided.

QoS differentiation in OBS networks is also an important issue. Certain types of QoS techniques applied in traditional store-and-forward electronic networks, such as active queue management, are no longer the best way to provide service differentiation in OBS unless we accept the loss of the optical data transparency. Thus, other types of techniques need to be analyzed.

In this paper we give insight into the performance of a lossless (in the core of the network) enhanced multiservice OBS MAC protocol with QoS. A contribution of this paper with respect to past approaches is the use of the MAC on more complex topologies such as mesh. The protocol supports a great number of services by means of a dual access scheme: a queuearbitrated (QA) and a prearbitrated (PA) burst transmission method, for connectionless and connectionoriented TDM OBS services, respectively. Differentiation among connectionless classes is provided by a multiqueue priority system along with a distributed queue-arbitrated channel access module. This system permits higher-priority bursts from a node to preempt lower-priority bursts from other nodes on the network and be placed and transmitted ahead in time. Connection-oriented services use the prearbitrated channel access, which can incorporate different slotscheduling algorithms. The proposed algorithms deliver diverse results depending on the network state and traffic distributions; hence a dynamic decisionmaking protocol may enhance the scheduler according to the type of service request, the present network utilization, and the current traffic distribution on the network. The protocol is named DAOBS, which stands for distributed access for optical burst switching.

The remainder of the paper is as follows. Section II reviews existent QoS schemes, recent multiservice architectures, and MAC protocols for OBS networks. In Section III, the proposed MAC protocol is introduced and its QoS enhancements and access modes are described in detail. Results through simulations are analyzed in Section IV, and finally Section V concludes this paper with the main contributions and results.

\section{BACKGROUND ON QOS, MULTISERVICE ARCHITECTURES, AND MAC FOR OBS}

In OBS, QoS differentiation can be achieved by using many different strategies in order to guarantee different quality parameters, although most of them are based on a per-class approach [5]. Additionally, in the per-class method, QoS parameters can be differentiated as absolute (a fixed quality in terms of a certain parameter) or relative guarantees (the quality of each class is qualitatively or proportionally guaranteed between classes). Offset-based, preemption-based, and restriction-based schemes are three of the main burst loss differentiation QoS strategies for OBS.

Offset-based schemes [6] rely on the fact that bursts with a greater offset time should have more time to search for free resources on the network and be scheduled for switching with higher probabilities than those bursts with a smaller offset time. Thus, highpriority bursts are given a greater offset. Although this scheme increases the delivery rate of highpriority bursts, it also increases the latency of them, and for this reason, it is not a feasible scheme when both delay and loss rate need to be guaranteed at a reasonable level.

In the preemption-based QoS scheme [7], highpriority bursts are able to take over (preempt) the resources taken by low-priority bursts, whereas lowpriority bursts can never preempt high-priority bursts. Hence, on average, high-priority bursts see more available resources, which results in a lower loss rate.

Resource restriction-based schemes exclusively reserve a subset of the available resources for highpriority traffic only. An example is wavelength grouping [8], which prereserves wavelengths for highpriority bursts that can only be used by them even if the wavelengths are available for lower-priority traffic. Intuitively, the more wavelengths are reserved for high-priority traffic, the smaller its blocking probability will be.

Regarding the provisioning of multiple services using a common optical networking infrastructure, recent works have proposed the use of polymorphous optical burst switching (POBS) as an efficient way to cope with the high-speed and optical transparency requirements of future optical networks. For instance, [9] proposes a common and integrated signaling system to provide an extensive number of services using POBS, whereas [10] gives an exhaustive analysis of the best-effort burst-based service performance when some channel capacity is allocated to TDM services. Nevertheless, neither of them provides a detailed and specific architectural protocol analysis to support POBS.

In terms of MAC protocols for OBS networks, these have been given little attention, and almost all existing studies are focused on OBS metro-ring networks [11]. For instance, [12] proposes a simple MAC protocol called beforehand bandwidth reservation for OBS ring networks to reserve the empty slots in the next big-slot cycle. Likewise, in [13] a loss-free OBS metro 
ring architecture (CORNet) is designed along with a distributed MAC protocol to integrate the support of differentiated service and fairness access. The proposed QoS provisioning mechanism adopts a bandwidth-reservation approach that combines realtime transmission establishment and termination routines. Furthermore, a credit-based fairness control scheme is defined to guarantee the transmission fairness of best-effort traffic. Recently, [14] has proposed a multiple-token-based MAC protocol for OBS ring networks using a tunable transmitter and one tunable receiver (TT-TR). In order to avoid or reduce the receiver contentions, tokens manipulate the wavelength accessibility and the destination queues decide on the burst scheduling.

The lack of MAC protocols on mesh network topologies and the need to provide integrated optical network architectures able to address the QoS and multiservice demands for the future optical Internet motivate the present work. Next, a detailed description of the MAC protocol and its operation follows.

\section{Multiservice QOS-ENABLED MAC FOR OBS}

In this paper we conceive an optical burst-switched network without wavelength conversion. Furthermore, the network data channel is time sliced, e.g., slotted OBS [15], and a constant-based offset scheme is used as in [16]. This last approach allows us to use fixed offsets by means of input delay lines at each input burst data port of a length equivalent to the maximum delay incurred in the processing of the burst control packet (BCP).

One of the main differences of DAOBS compared with other MAC-based schemes for OBS networks is that, in the former case, the protocol runs on mesh network topologies. To realize this, the network is logically partitioned in monocolor light-trees. Conceptually, the DAOBS optical tree has some similarities to a light-trail [17]. Both propose network architectures that can span over ring and mesh topologies using optical buses/lightpaths enabling the participating nodes to share the capacity of the channel in a spatial reuse time-shared basis. Moreover, both also define the figure of a controller, the head of bus (HoB) in DAOBS, and the arbitrator in the light-trail. However, DAOBS is also a burst MAC protocol with collision avoidance in the core of the network that ensures the delivery of the burst without using electronic buffers, as in [17]. Furthermore, the request-granting process operates differently. In the DAOBS QA access mode this process works as a very simple algorithm based on a counting and monitoring process, whereas in the light-trail solution an explicit request grant is established between the client node (the node requesting slots) and the arbitrator, which decides what node is granted in a per-slot basis. Finally, DAOBS also includes a wavelength assignment module since a node can belong to more than a single light-tree, and therefore multiple path/wavelength options are available to the nodes while transmitting data.

Despite that the analysis of the different methods to instantiate light-trees is out of the scope of this paper, it is worth mentioning that for the evaluation of the present protocol a greedy metaheuristic graphcoloring algorithm has been used. This algorithm is based on the coloring of the graph using minimumspanning trees and the remaining uncolored links, altogether conforming a superset of the set of light-path virtual topologies [18] able to improve the utilization capacity of the network. One of the advantages of this coloring scheme is that through this tree-based instantiation, collisions from two different input ports are avoided, which is especially useful in the case of dealing with OBS networks without wavelength conversion. Furthermore, light-tree-based topologies are useful for delivering multicast or groupcast services or merely for multimedia broadcasting. Besides, optical musticasting can be more efficient than electronic multicasting since "splitting light" is conceptually easier than copying a packet in an electronic buffer.

The root or topmost node of the light-tree is the head-of-bus node, and all leaf nodes are tail-of-bus (ToB) nodes (see Fig. 1). Under this scenario, we consider two unidirectional control channels, which can be in-fiber (i.e., using a specific wavelength): the downstream or forward channel, which goes from the HoB node to the ToB nodes, and the upstream or reverse channel, on which $\mathrm{QA}$ access request packets are forwarded from the ToBs to the HoB. The HoB node is responsible for generating and forwarding the BCPs in the DAOBS light-tree at every time slot. The light-tree is normally composed of other nodes between the HoB and ToB. According to the operation of the different MAC access modes, all the nodes can

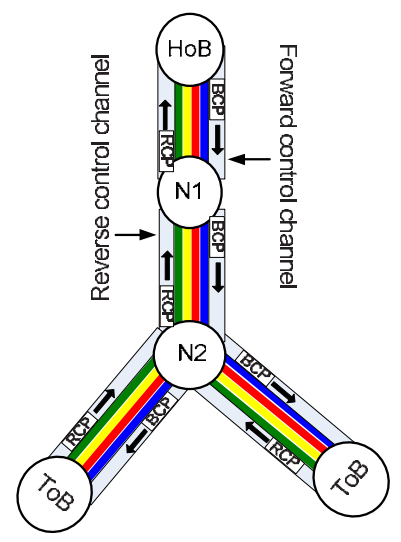

Fig. 1. (Color online) Example of a DAOBS light-tree. If node N1 needs to transmit to any ToB, it requests for free slots to the $\mathrm{HoB}$ or eventually waits for a free slot. 
transmit bursts to the rest of the downstream nodes making use of the multiplexing capabilities within the light-tree.

As has previously been introduced, every node can belong to manifold light-trees. As a result, a node may have to manage multiple DAOBS entities, one for each accessible light-tree. In the proposed architecture, a DAOBS entity is identified by its input port +output port + wavelength, and a DAOBS light-tree by its $\mathrm{HoB}+$ wavelength on the network.

DAOBS provides two channel access mechanisms: queue arbitrated and prearbitrated. Each access scheme uses a different slot type. The former is devised for connectionless burst transport services, that is, burst transmissions that have not been explicitly acknowledged about the availability of a certain channel capacity. On the contrary, PA is used by services or applications that need a guaranteed reserved bandwidth. For a more detailed application usage example we refer to Table III in the results section. Figure 2 shows an example where bursts of different service types are transported on three wavelength channels. As seen in the figure, connectionless bursts are transmitted in QA slots, sharing the available channel capacity together with connection-oriented applications making use of PA slots. The number of PA reserved slots depends on the application bandwidth requirements. For instance, on channel $\lambda 3$ two consecutive slots (every six) are used by a single connection.

In order to support such an architecture of services, the burst control packet has a 1 bit field (PA/QA) to announce the upcoming slot type as shown in Fig. 3. If this field is equal to 1 , the network node currently processing the BCP runs the PA access mode. Otherwise, the node executes the QA access mode. BCPs are created at the $\mathrm{HoB}$ nodes every time slot, transmitted over the control channel and processed by the core nodes in advance to the reception of the upcoming slot (e.g., eventually taken by a burst). The PA/QA type is assigned according to the flow diagram from Fig. 4. The HoB always (i.e., at every slot) creates QA slots

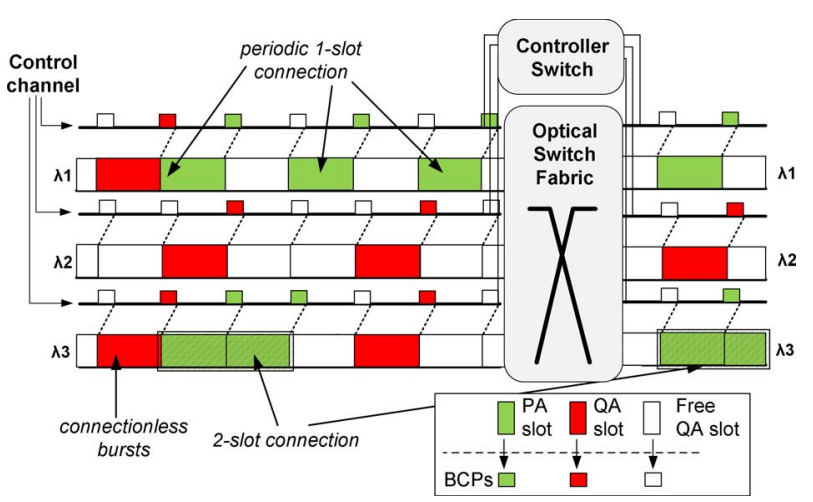

Fig. 2. (Color online) Data channel PA/QA slot example.

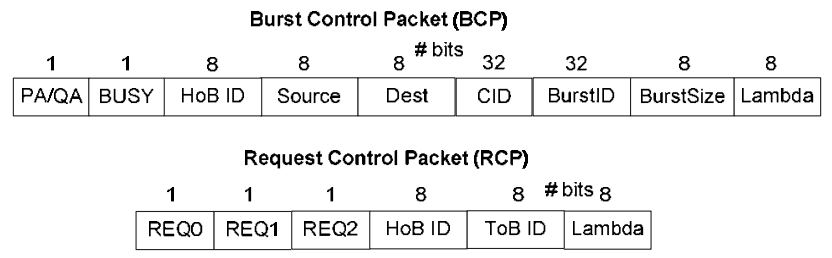

Fig. 3. BCP and RCP packet formats.

unless a PA slot has been reserved by the service layer on the slot supercycle window. In such a case, a new PA slot is created by setting the PA/QA field to 1. Otherwise, a QA slot is forwarded to the next node downstream on the light-tree. In both cases, the HoB checks whether it is indeed able to fill the next slot by transmitting one of its enqueued bursts.

Figure 5 shows a high-level diagram of the multiservice-aware DAOBS architecture. The OBS MAC is composed of four main submodules: the QA and PA burst access modules; the slot scheduling (SS) algorithm module (only in the HoB); and the burst assembly, classification, and scheduling module. This last module includes the burst assembly algorithms and classifies the burst between the QA and the PA modules according to its type.

\section{A. Queue-Arbitrated Access}

In the QA access, the two unidirectional control channels transport the BCPs and RCPs: on the downstream or forward channel, the BCPs are forwarded from the HoB node to the ToB nodes, and on the upstream or reverse channel, the RCPs are forwarded from the ToBs to the HoB. As has already been introduced, both types of control packet are sent every time slot. This is necessary in order to allow all the nodes participating in the light-tree to check whether free slots are coming and to permit them to request free slots periodically. Since the bursts are much longer than the packets, the electronic processing requirements can easily be met.

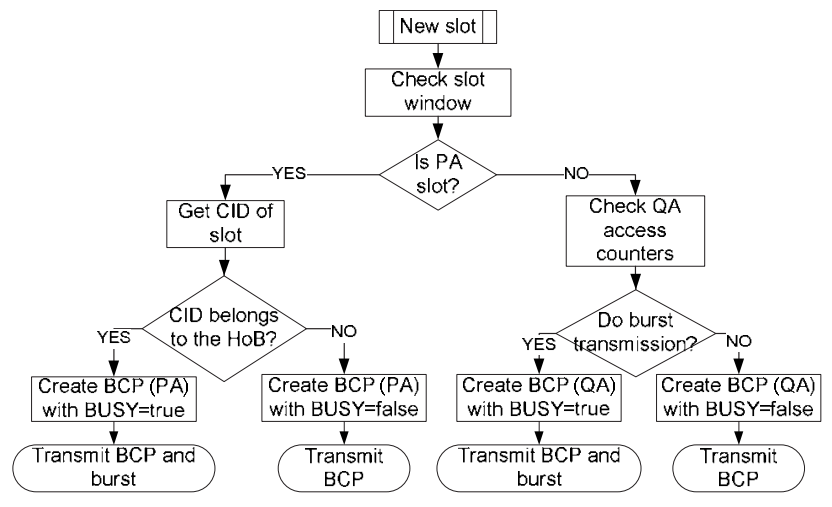

Fig. 4. Flow diagram at the $\mathrm{HoB}$ slot processing. 


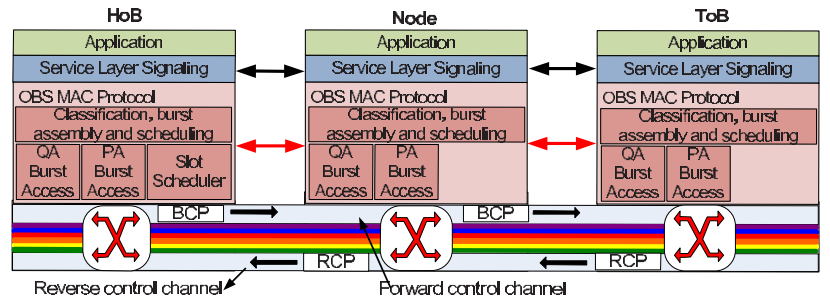

Fig. 5. (Color online) DAOBS protocol and network architecture.

All the nodes in the light-tree can transmit bursts to downstream nodes according to the operation of the QA access, that is, a node requests free slots to the upstream and HoB nodes using the REQ bits of the RCP to subsequently transmit bursts by taking the upcoming free slots on the downstream direction. A set of counters for each priority keeps track of the requests and free slots coming from downstream and upstream nodes, respectively. Connectionless burst losses only happen at the edge of the network due to queue blocking when more load is offered than the acceptable.

BCPs have a BUSY bit for announcing whether the upcoming slot is taken up, whereas RCPs have a REQ bit for each burst priority class. Figure 3 shows an example of the packet format for both the BCP and RCP with three priority classes. Recall that a QA access slot is announced by the PA/QA field set to 0 . In the case that the BUSY bit is equal to 1 , then the rest of the fields in the BCP (BurstID, Source, Dest., etc.) are meaningful since the upcoming slot is taken.

The QA access module of the DAOBS MAC entity in a node is composed of the following components for each priority class $i$ : a distributed access state machine (DASM), a request control machine (RQM), a local queue (LQ), and a distributed queue (DQ). Figure 6 depicts the relation between all these elements for a DAOBS entity on wavelength $\lambda_{k}$ with three burst pri-

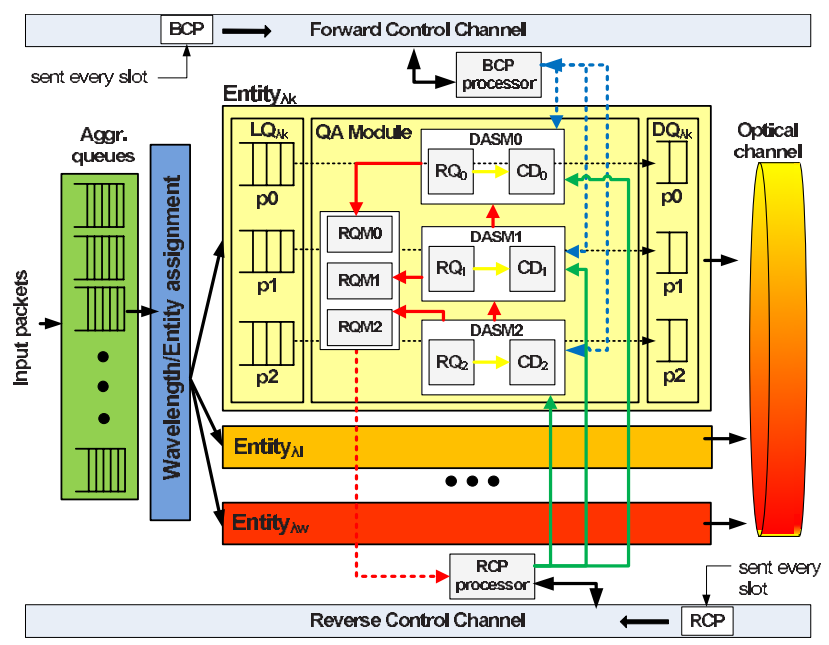

Fig. 6. (Color online) QA entity with three burst priorities on wavelength $\lambda_{k}$. ority classes, and the interconnection between the control, state machines, and packet processors.

The LQ temporarily stores the bursts from the wavelength/entity assignment module while waiting to gain access to the channel. The DQ is a one-position queue that stores the next burst to transmit. The set of DQs from the nodes that belong to the DAOBS light-tree forms the so-called virtual distributed queue. Thus, when a burst at a certain node gets into one of its DQs, it is like accessing a first in, first out (FIFO) queue distributed among the nodes from the light-tree. Furthermore, at each priority there is also a RQM that monitors the requests triggered for that priority. Finally, the DASM is responsible for monitoring and managing the counting process of the protocol. There is a DASM for each priority, and in the example given in Fig. 6 these are DASM0, DASM1, and DASM2, where the greater the number, the higher its priority. As shown in the figure, high-priority DASM can signal and preempt lower-priority DASMs to ensure that higher-class connectionless bursts receive a better service level.

Each DASM can be in two different states as shown in Fig. 7. In the idle state, the OBS node, for a certain DAOBS light-tree and priority, has nothing to transmit, whereas in the active state, the node has successfully made a request for a free slot and is waiting to transmit the burst using that slot. Apart from this, each DASM has two counters: a request counter (RQ) and a countdown counter (CD). While the RQ monitors the number of requests made by downstream nodes on the optical light-tree and by higher-priority DASMs in its own DAOBS entity, the CD counts the number of free slots the current node is not allowed to use before being given access to transmit a burst. All entity counters are initialized to 0 .

While a DASM at priority $i$ is idle, it monitors the RCPs on the reverse control channel and BCPs on the forward control channel and increases [see Fig. 7 step

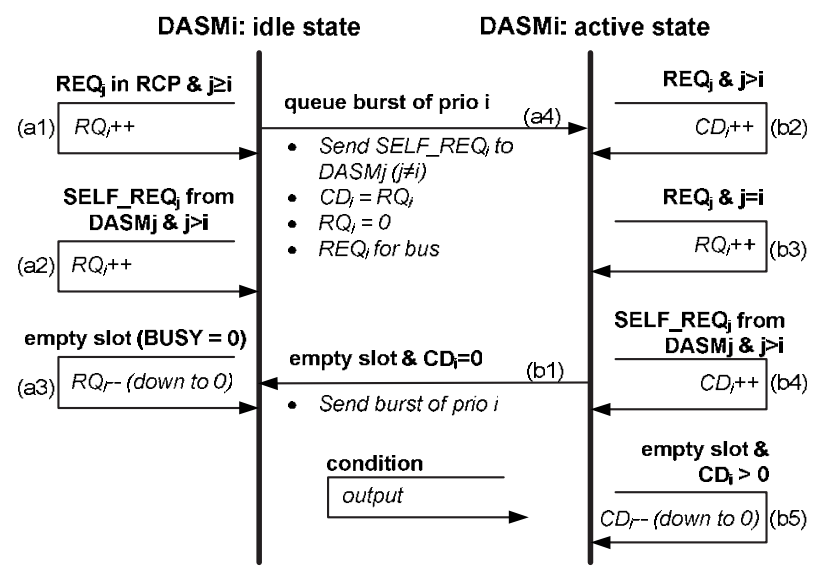

Fig. 7. QA DASM flow at priority $i$. 
(a1)] or decreases (a3) the $R Q_{i}$ for every $R E Q_{j}=1$ in the RCP of a priority $j \geqslant i$ and for every $B U S Y=0$ in the BCP, respectively. Similarly, if the DASMi receives a $S E L F \_R E Q_{j}$ signal from a higher-priority DASMj $(j>i)$ (a2), then the $R Q_{i}$ is also increased.

As soon as a burst is stored in the LQ of a certain DAOBS entity and priority $i$, if the DASMi is idle it switches to the active state following the transition (a4) shown in Fig. 7. The same happens if after returning from a successful burst transmission there are more bursts to transmit in the LQ. This state transition (a4) triggers the following events: first, a $S E L F \_R E Q_{i}$ signal is sent to the remaining DASMj $(j \neq i)$ in the entity; then the value of the $R Q_{i}$ is dumped to the $C D_{i}\left(R Q_{i} \leftarrow C D_{i}\right)$, and the $R Q_{i}$ is then reset to 0 ; and finally a $R E Q_{i}$ signal is sent to the $\mathrm{RQM}$ of that same priority in order to set the REQ bit of that priority to 1 in the next upcoming free RCP received from the downstream node.

In the active state, the DASMi continues monitoring the BCPs and RCPs. For any REQ bit of priority $j>i$ (b2) or $S E L F \_R E Q_{j}$ signal from a DASMj with $j>i$ (b4), $C D_{i}$ is increased by 1 . These two steps let higherpriority bursts on the DAOBS light-tree be placed ahead of and thus transmitted before, even between bursts from two different nodes of the same tree. In (b3), for every REQ bit of priority $j=i$, the $R Q_{i}$ is increased by 1 . Likewise, for every empty slot on the forward control channel (b5), and provided that the $C D_{i}$ $>0$, the $C D_{i}$ is decreased by 1 (down to 0 ). Finally, the transmission of the burst from the DQ at priority $i$ happens when $C D_{i}$ reaches 0 and an empty slot comes from the upstream link. This last step involves the transition (b1) of DASMi from active to idle.

The wavelength assignment is responsible for assigning burst transmissions to a specific DAOBS light-tree. As stated before, each DAOBS tree is identified by its $\mathrm{HoB}$ and wavelength. Therefore, the wavelength selection is in fact a DAOBS entity assignment (see Fig. 6). In the QA access, the wavelength assignment is controlled by an algorithm that takes into account the values of the counters RQ and CD and the number of bursts ahead in the LQ. As we have previously introduced, the value of these counters determines the position of the node in the distributed FIFO queue at a certain priority class. The number of bursts in the LQ gives information about the number of transmissions not processed yet. Depending on these values, the node will take longer to transmit a burst on that specific light-tree, thus increasing or decreasing the channel access delay, and consequently, the end-to-end delay of the burst. In this process, the DAOBS light-tree that is expected to provide the lowest access delay is always selected. That is, for priority $i$

$$
\min _{\lambda_{j} \in \mathcal{W}_{n}^{m}}\left[R Q_{i, \lambda_{j}}+C D_{i, \lambda_{j}}+\operatorname{size}\left(L Q_{i, \lambda_{j}}\right)\right],
$$

where $\mathcal{W}_{n}^{m}$ is the wavelength/DAOBS candidate list for transmitting a burst from node $n$ to $m$.

One of the advantages of the proposed protocol is its low complexity. In the QoS-enabled DAOBS QA access mode, the hardware complexity is easily bearable. Furthermore, the scheduling complexity is again virtually null as the QA mode just works as a set of counters that can easily be monitored and updated.

\section{B. Prearbitrated Access}

In the PA access, downstream (core) nodes explicitly request the connection setup to the light-tree HoB for a guaranteed channel bandwidth (periodic transmission of bursts). In turn, the $\mathrm{HoB}$ acknowledges the core node about the success of the connection. Although this feature is supported by a higher service layer that monitors applications' service requests, it is the MAC layer that implements the scheduling, allocation, and differentiation of slots. Service layer messages are transmitted and forwarded through the control channel together with the BCPs and RCPs.

Figure 8 shows an example of an explicit multilayer setup and release of a TDM connection-oriented channel and the slot allocation at the MAC level. Let a user/application request a connection channel between node $\mathrm{N} 1$ and a downstream node on the light-

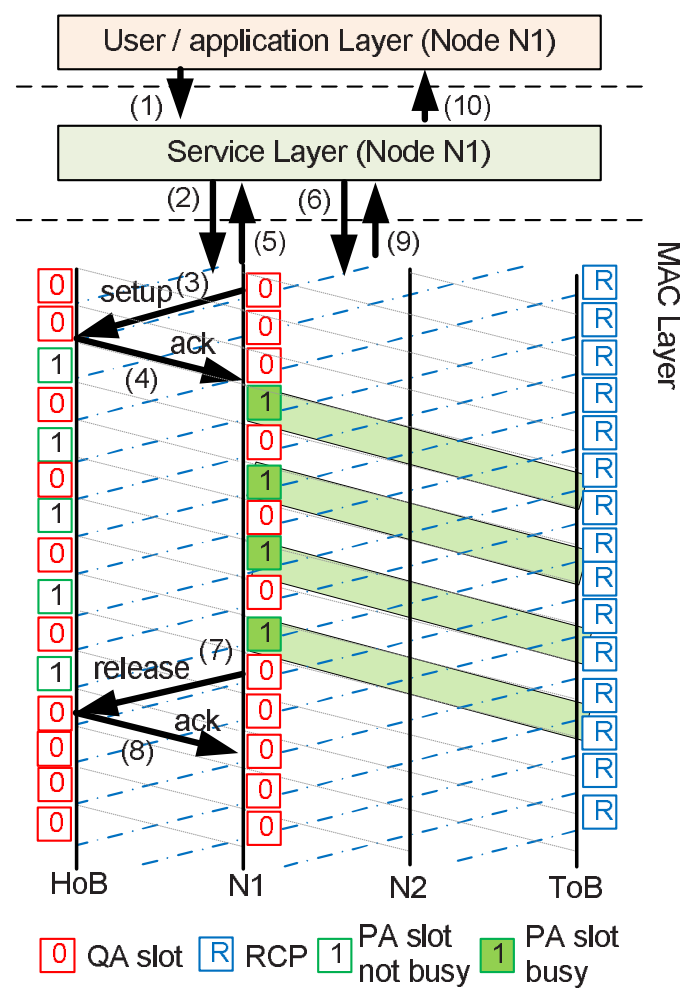

Fig. 8. (Color online) Example of a multilayer PA service setup and allocation of slots in the MAC layer. 
tree (1) (e.g., the ToB). The service layer computes the connection requirements of the application call (2). For instance, let the service layer at node N1 map the connection to use 1 out of 2 slots, as shown in Fig. 8 . At this step, the service layer also sorts the list of possible HoBs that can serve this service. Using this algorithm, the setup message is transmitted to the chosen $\mathrm{HoB}$ that is in charge of scheduling all the PA slots for this DAOBS light-tree (3). Upon receiving the setup message, the HoB allocates, if possible, the slots for the connection from N1. It is worth noting that many different scheduling algorithms can be implemented in order to satisfy a variety of service requirements, such as, jitter, delay, bandwidth, call blocking probability, etc. After processing the slot scheduling, the HoB acknowledges the node whether the connection has been scheduled or not by means of an acknowledgment message (4). The service layer at N1 is notified about the availability and successful setup of the connection (5), in which case, the application can start transmitting its data packets. The transmission of bursts will be made according to the PA reserved slots allocated to node N1. When the service finishes, the service layer initiates the disconnection (6) by sending a release message to the $\mathrm{HoB}$ node (7), which liberates the allocated slots for the connection. An acknowledgement message is then transmitted back to node N1 (8) to inform the service layer of the successful release of the connection (9). An explicit acknowledgment signal can also be sent to the application layer (10).

One of the advantages of the DAOBS PA slot allocation is that even using an explicit release, eventual upcoming PA slots that would remain unused can be changed by the designated core node and announced as a QA slot to the rest of the downstream nodes. For instance, in Fig. 8, the node N1 initiates the release of the connection sending a message to the HoB. Meanwhile, a PA slot has already been created and scheduled at the HoB following the information stored in the connection table, which is not updated yet due to the message propagation delay. In such a case, if the PA slot belongs to a connection from N1, and the connection is in the release state, the node can change the type of slot to be used by other downstream nodes or even reuse it to transmit one of its bursts through the QA access.

In order to reduce the connection establishment delay, a cross-layer algorithm in the service module sorts the list of HoBs available to serve the TDM connection so that light-trees for whose own service request node is actually a $\mathrm{HoB}$ are given preference. As a result, the setup can be processed between the service and MAC layers in the node itself avoiding a two-way connection establishment, hence reducing the connection setup delay.
Along with the PA channel access, the MAC layer in the HoB nodes is enhanced with a slot scheduling manager for allocating the connections for all its downstream nodes, including itself. The allocation of slots is made along a supercycle slot window of a size that depends on the minimum and maximum possible bandwidth request. For instance, with a $10 \mathrm{Gbps}$ channel and a minimum connection request of $155 \mathrm{Mbps}$, equivalent to an OC-3 of a synchronous optical network (SONET), the size of the supercycle window will be

$$
W=\left\lfloor\frac{10 \times 10^{9}}{155 \times 10^{6}}\right\rfloor=64 \text { slots . }
$$

With regard to the specific scheduling algorithms, Fig. 9 shows three different algorithms for a 4-slot TDM connection: (1) a request for 4-slot service over first-fit consecutive slots (FF); (2) a request for 4-slot TDM over a periodic number of slots (SPFF); or (3) an allocation of a 4-slot bandwidth guaranteed service over a number of slots not necessarily periodic or consecutive, but random (NCR). FF-based algorithms can be considered in order to support not only applications with guaranteed bandwidth requirements, but also with minimum delay variation between supercycle periods. Periodic scheduling is also necessary to provide jitter-controlled characteristics for other multimedia services with periodicity within the supercycle.

The periodic scheme is implemented by the sliding periodic first-fit (SPFF) algorithm. In SPFF, the number of requested slots $N$ must obey that $\operatorname{size}(W)$ $\equiv 0(\bmod N)$, where $\operatorname{size}(W)$ is the size of the slot window $W$ (in number of slots). Equation (3) represents mathematically the set of slots that guarantee the constraints imposed by the SPFF algorithm. The scheduling is successful if a group of slots $S_{i}$ can be found such that each slot $s_{i}$ is free $\left[v\left(s_{i}\right)=0\right]$ in the window fulfilling the periodicity constraint, $t=W / N$ :

$$
S_{i}=\left\{s_{i} \in W \mid v\left(s_{i}\right)=0 \forall s_{i+1}=s_{i}+t, t=\frac{W}{N}, 1 \leqslant i \leqslant N\right\} .
$$

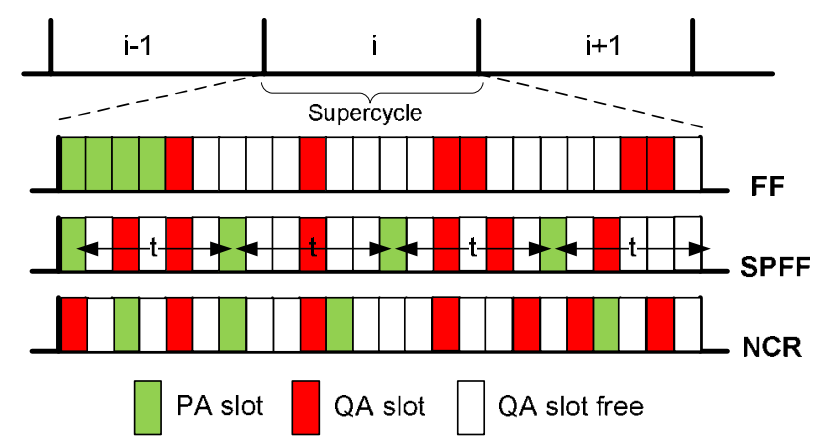

Fig. 9. (Color online) PA slot scheduling algorithms. 
Figure 10 shows in detail the SPFF algorithm. In the algorithm pseudocode, the input $S_{w}$ stands for the predefined amount of sliding slots for every execution of the algorithm; $S_{0}$ is the last reserved slot from the previous algorithm invocation; and $s_{p}, s_{s}$, and $s_{j}$ are auxiliary pointers used by the algorithm to check the free slots that fulfill the constraints from Eq. (3). Line (6) captures the last reserved slot used in the previous invocation of the algorithm and adds to it a number of slots $\left(S_{w}\right)$ so that the initial slot to be checked moves ahead (slot sliding). From that point, the availability of free slots is checked for the number of slots $N$ passed as a parameter to the algorithm until the group of slots, $S_{i}$, is found. Depending on $N$, the number of iterations $(t)$ in the while loop changes, hence the smaller the number of requested slots $N$, the greater the possibilities to find $S_{i}$.

One of the benefits of this algorithm is that, not only does it accomplish the periodicity for the slot scheduling within the slot window, but it also enhances the success rate for $n$-slot connections. Now the connections demanding very few slots, $m$ (where $n>m$ ), are allocated along the slot window reducing the collision with the required space demanded by other $n$-slot connections.

\section{RESULTS}

This section analyzes the performance of the multiservice QoS-enabled MAC protocol proposed in this

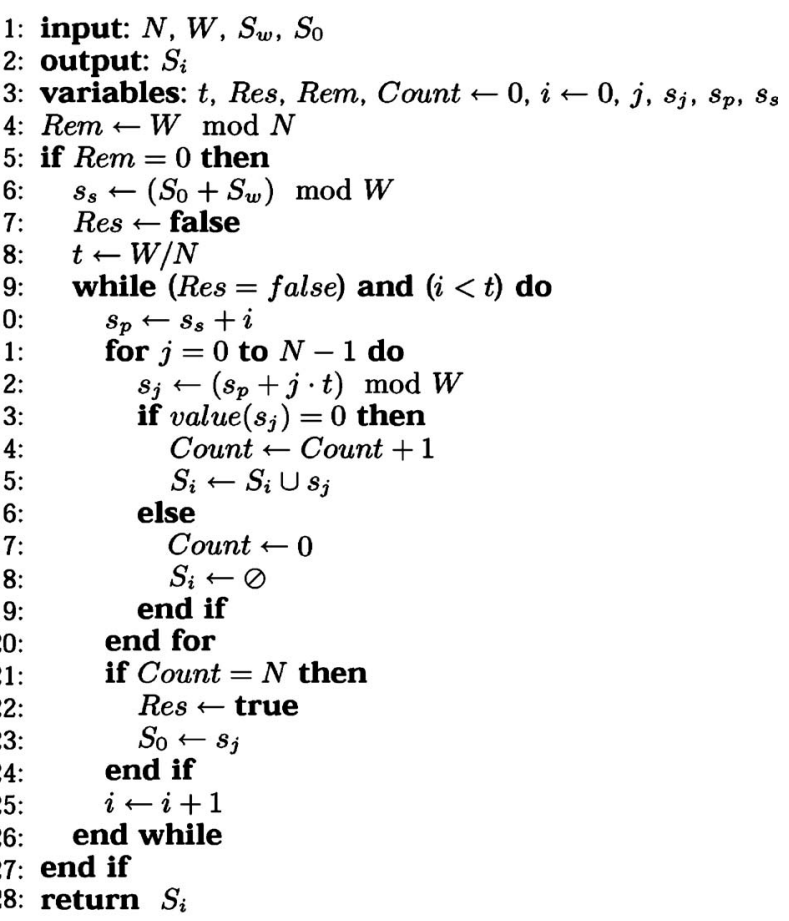

Fig. 10. SPFF scheduling algorithm. paper. To this end, simulations are conducted on the well-known NSFNET network composed of 14 nodes and 21 bidirectional links. In such a scenario, there are 16 wavelengths with 10 Gbps per channel. We assume in all the examples that the network neither has wavelength converters nor FDLs for contention resolution, hence burst transmissions are subject to the wavelength continuity constraint. Regarding the setup of hardware devices, the control packet processing time and the nonblocking matrix switching time are set to 10 and $5 \mu \mathrm{s}$, respectively.

With respect to the traffic characteristics, bursts are created at each node by using a volume-based algorithm [19] with an input packet arrival Poisson process and fixed size per burst of 100,000 bytes. For simplicity, the burst destination is uniformly distributed to all the remaining nodes, so that the probability of a burst being sent to any other node in the network is the same.

Results are gathered using the batch means method. 95\% confidence intervals were also obtained, but since they are quite narrow, they have been omitted in order to improve the readability of the graphs.

The results section is divided into four main subsections. First, insight into the performance of the QA access mode as a function of the local queue size and without QoS is provided. Second, an analysis of the QA access mode with QoS is presented. The next subsection evaluates the performance of the whole MAC architecture with both connection-oriented and connectionless burst services. Finally, a critical analysis about the proposed service classification is presented.

\section{A. Results of the QA Access Mode Without QoS}

The initial results deal with the protocol performance of the QA access mode when different local queue (LQ) sizes are used and only one connectionless traffic class is transmitted on the network. Figure 11(a) shows the burst blocking probability (BBP) as a function of the total offered load to the network in erlangs per wavelength (Er/wl). The LQ sizes (in number of bursts) used throughout the simulations are 2, 5,100 , and 1000 bursts. Intuitively, the smaller the size of the LQ, the sooner the blocking probability starts rising. For sizes between 2 and 100 bursts, the results at high loads asymptotically converge to the same value. Only for the case in which the LQ size is equivalent to 1000 bursts can we see an improvement $(\sim 50 \%)$ of the blocking probability but at the expense of increasing its size by nearly 2 orders. Furthermore, Fig. 11(b) represents the mean access delay (in $\mathrm{ms}$ ) for the same group of LQ lengths. At the expense of decreasing the mean blocking probability, the delay experienced when the LQ size is of 1000 bursts rises up to nearly $30 \mathrm{~ms}$ at very high loads. Thus, a trade-off 

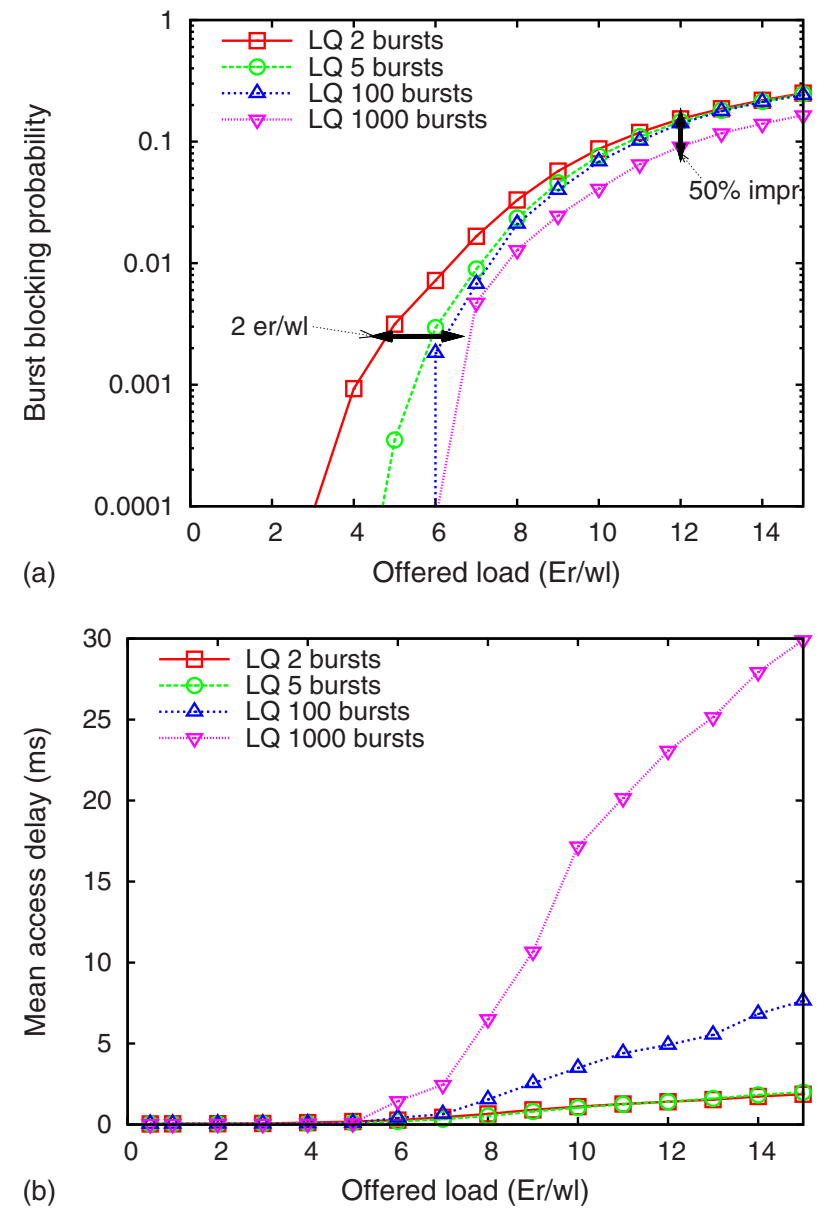

Fig. 11. (Color online) QA access mode without QoS for different LQ lengths: (a) burst blocking probability, and (b) mean access delay.

between $\mathrm{BBP}$ and access delay comes up. At low-tomedium offered loads, the BBP improvement for larger LQ is considerable. However, at high loads, an average access delay 15 times greater may not justify a little BBP improvement of about 50\%.

\section{B. Results of the QA Access Mode With QoS}

In this section, we give insight into the results of the DAOBS QA access mode when a number of different connectionless burst traffic classes are transmitted on the network. In this experiment the LQ size is limited to 5 bursts. The following notation is used in the graphs: burst priority classes are numbered from 0 to 2, with class 2 being the highest-priority traffic. To demonstrate the performance of the protocol on different traffic scenarios, two traffic class distributions have been tested, as shown in Table I.

Figure 12(a) shows the BBP as a function of the offered load in the two traffic distributions. Class 2 blocking probability is not plotted on the graph because in both cases it is null for the whole load range. The rest of classes get different results when changing
TABLE I

TRAFFiC Distribution Configurations

\begin{tabular}{cccc}
\hline Distribution & Class 0 & Class 1 & Class 2 \\
\hline 1 & $50 \%$ & $30 \%$ & $20 \%$ \\
2 & $70 \%$ & $20 \%$ & $10 \%$ \\
\hline
\end{tabular}

the traffic configurations. Besides, we can see in the second distribution that when the higher-priority traffic load is decreased with respect to the total, the burst blocking probability decreases for both class 0 and class 1 . Intuitively, the lower the class 2 (i.e., the highest priority) traffic load, the more resources available for the rest of the classes. Consequently, less high-priority traffic preempts lower-priority traffic; hence the low-priority traffic loss probability decreases. Nevertheless, at high load the mean BBP plots of both traffic distributions tend to converge to similar values, which ensures a predictable average $\mathrm{BBP}$ performance of the protocol whatever traffic distribution is present on the network.

Figure 12(b) compares the mean access delay as a function of the offered load. In both traffic configurations, class 2 bursts not only have the lowest access delay, but also get a delay nearly constant for the whole load range. Class 0 bursts have a similar delay trend for both traffic configurations, and, finally, class 1 bursts (i.e., the intermediate class) get a different access delay depending on the traffic distribution. When the class 2 proportion is $10 \%$ and class 1 represents $20 \%$ of the total traffic on the network, the access delay for class 1 resembles more the delay of class 2 . Thus, it can be concluded that the delay experienced by a burst traffic class depends on the aggregate traffic between itself and all its higher-priority traffics.

Figure 12(c) shows a different performance parameter. In the graph, the probability that a certain class of traffic is being transmitted from a HoB node is counted. Clearly, we can observe that class 2 bursts are mainly transmitted from $\mathrm{HoBs}$, whereas the transmission rate for class 0 and class 1 decreases almost linearly as the load is increased. High-priority burst traffic is more often transmitted from a HoB because this node tends to see the capacity channel of the network as more available while the requests from downstream nodes are not yet received due to propagation delays.

Figure 12(d) shows the mean route length (in number of hops) for the three burst priorities. As can be seen in the graph, the highest-priority bursts almost have a constant route length for the entire load range. Nonetheless, the lowest-priority bursts experience a drop in the path length that is directly related to the fact pointed out in Fig. 12(c). Moving class 0 burst 

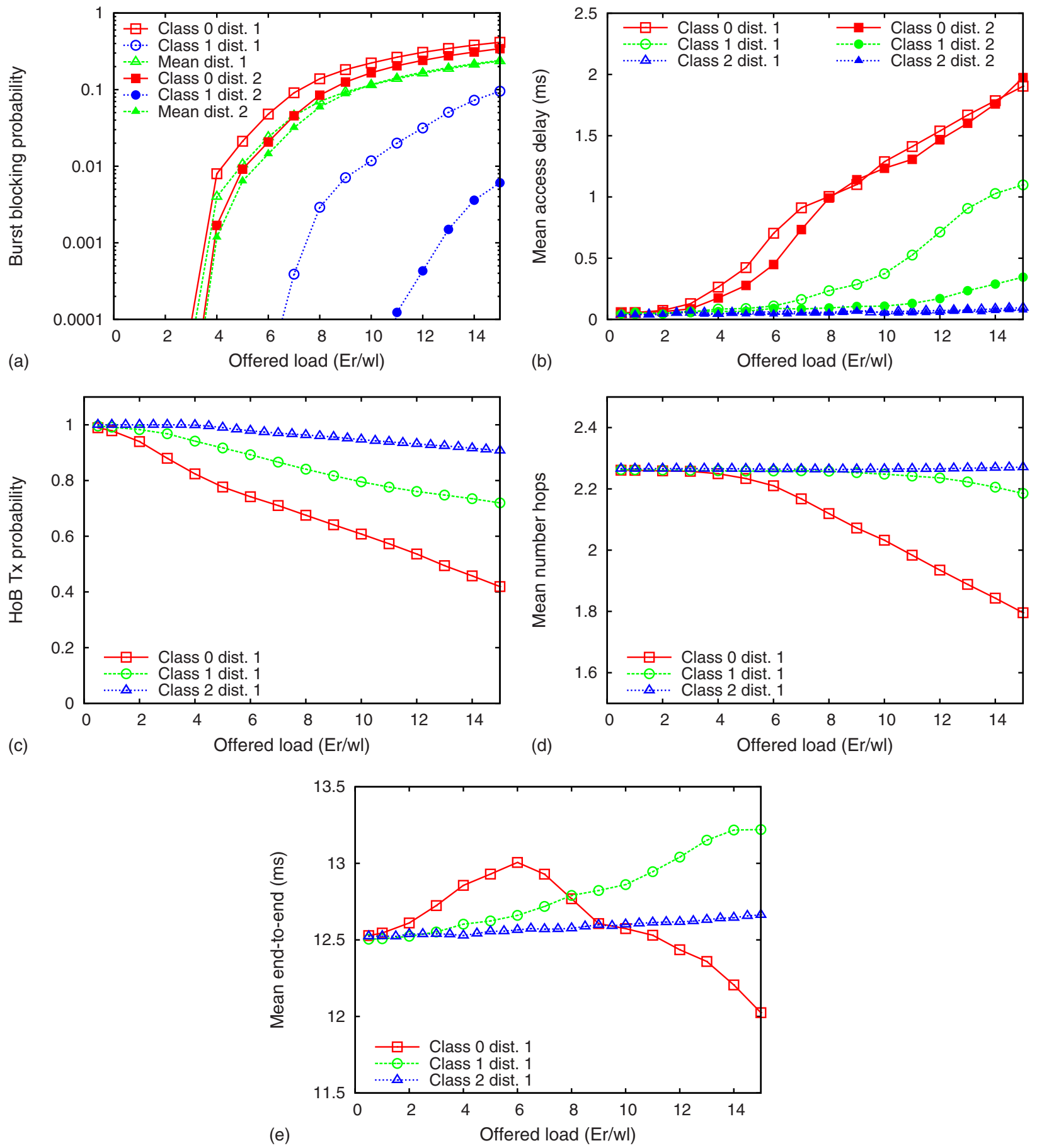

Fig. 12. (Color online) QA access mode with QoS and LQ=5 bursts: (a) burst blocking probability comparison between the two traffic distributions, (b) access delay, (c) HoB transmission probability distribution 1, (d) mean number of hops distribution 1, and (e) end-to-end delay distribution 1 .

transmissions from the $\mathrm{HoB}$ to inside the DAOBS light-tree brings the origin node closer to the destination, which at most can be one of the ToBs, and therefore the route is shortened.

With respect to the burst end-to-end delay (in $\mathrm{ms}$ ), Fig. 12(e) shows a comparison between the three classes. In this specific scenario, an average end-toend delay between $1.5 \mathrm{~ms}$ is kept between the three traffic classes still providing a clear differentiation of burst loss probability. Specifically, class 0 bursts see an increase in their end-to-end delay as the offered load rises, according to the access delay increase in Fig. 12(b). However, we can see a trend change from an offered load of $6 \mathrm{Er} / \mathrm{wl}$ onwards where the delay starts falling. Following the reasoning from previous figures, this fact can be explained as follows: at high loads, class 0 bursts get a higher blocking probability and those that are able to get to a destination tend to 
follow a shorter path, i.e., fewer number of hops, hence decreasing the mean delay even though the access delay goes up as shown in Fig. 12(b). The rest of the traffic classes (class 1 and class 2) experience an end-to-end delay only affected by the access delay increase since both of them show almost a constant mean route length within the load range under consideration [see Fig. 12(d)].

In order to evaluate the performance not only in terms of the offered traffic load, but also as a function of the carried traffic load, Fig. 13 shows the blocking probability as a function of the average network load ratio $\rho=\sum_{i=1}^{n} M_{i} / \sum_{i=1}^{n} C_{i}$, where $M_{i}$ is the traffic carried by link $i, C_{i}$ is the capacity of link $i$, and $n$ is the number of links (bidirectional) on the network. The network load is represented between 0 and 0.5 and computed using the link utilization results from the same simulation runs. As in Fig. 12(a), class 2 is lossless for the considered network load; hence it is not represented in the graph. For the rest, the protocol provides differentiation of up to three orders of magnitude between class 0 and class 1 at a load of 0.3 , and an improvement of 2 orders for class 1 traffic between the two distributions at load 0.45. Based on the QoS requirements shown in Table II and providing that the packet loss rate (PLR) can be approximated by the BBP for fixed size bursts, the protocol can guarantee the QoS of a diverse number of applications. High or very high loss sensitive traffic (e.g., grid applications or live video broadcasting) can be mapped as class 1 for a great network load range (up to $45 \%$ for the second traffic distribution) or even be mapped as class 2 traffic, which is loss-free.

\section{Results With Mixed Connectionless and} Connection-Oriented Burst Services

This subsection analyzes the network performance of the proposed OBS MAC protocol using the three

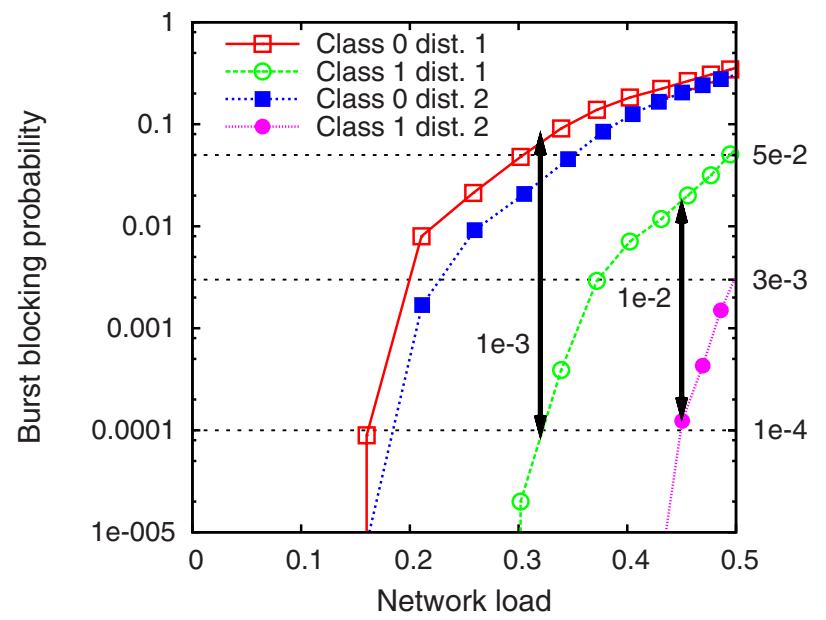

Fig. 13. (Color online) QA access mode BBP as a function of the network load.
TABLE II

APPLICATIONS' QOS REQUIREMENTS [20]

\begin{tabular}{llll}
\hline Application & Delay & Jitter & $\begin{array}{c}\text { Loss sensitivity } \\
\text { (PLR) }\end{array}$ \\
\hline $\begin{array}{c}\text { Interactive } \\
\text { audio/video }\end{array}$ & $<150 \mathrm{~ms}$ & $<75 \mathrm{~ms}$ & High $(<1 \mathrm{e}-3)$ \\
$\begin{array}{c}\text { Interactive } \\
\text { transaction } \\
\text { data }\end{array}$ & $<50 \mathrm{~ms}$ & $<10 \mathrm{~ms}$ & $\begin{array}{c}\text { High }(\text { game }<5 \mathrm{e}-2) \\
\text { to very high } \\
\text { (grid }<1 \mathrm{e}-4)\end{array}$ \\
$\begin{array}{l}\text { Video/audio } \\
\text { streaming }\end{array}$ & $<2 \mathrm{~s}$ & $<40 \mathrm{~ms}$ & $\begin{array}{c}\text { High }(<3 \mathrm{e}-3) \text { to very } \\
\text { high }(\text { live video } \\
<1 \mathrm{e}-4)\end{array}$ \\
$\begin{array}{c}\text { Legacy } \\
\text { applications }\end{array}$ & Not & Not & Low \\
\hline & & specified & \\
\hline
\end{tabular}

aforementioned slot scheduling algorithms in a multiservice scenario where connectionless bursts share the capacity of the channel with connection-oriented guaranteed bandwidth services (TDM). Regarding the traffic characteristics, bursts are of fixed size for both types of service (connection-oriented and connectionless) and equal to the slot size $(100 \mathrm{kB})$. For the TDM services, we consider two different connection types of $155 \mathrm{Mbps}$ (S-155) and $622 \mathrm{Mbps}$ (S-622), equivalent to a bandwidth capacity of OC-3 and OC-12, respectively. The former is mapped to use 1 slot every 64 , and the second to use 4 out of 64 slots. In both cases, the connection call arrivals follow a Poisson process with an exponentially distributed holding time of 100 and $200 \mathrm{~ms}$, respectively (intentionally short to speed up simulations). The average load generated by the connection-oriented calls is calculated as follows:

$$
A_{C O}=\frac{1}{C} \sum_{i=1}^{n} \lambda_{i} \frac{1}{\mu_{i}} b_{i}
$$

where $C$ denotes the channel capacity in bps, $\lambda_{i}$ is the call arrival rate of connection service $i, 1 / \mu_{i}$ is the mean holding time, and, finally, $b_{i}$ is the demanded bandwidth of service $i$ in bps. Finally, two different traffic distributions have also been considered; first, a distribution in which TDM services represent $20 \%$ of the offered load and connectionless bursts the remaining $80 \%$ (Dist. 20\%-80\%), and second, a distribution with $40 \%$ and $60 \%$ (Dist. 40\%-60\%), respectively.

Figures 14(a) and 14(b) show the mean burst/call blocking probability of both service types (bursts and TDM) as a function of the offered load to the network in $\mathrm{Er} / \mathrm{wl}$ and distributed by the scheduling algorithm. In Fig. 14(a) we can see that the BBP is very similar for the three simulated scheduling algorithms, especially at high loads, where the three converge to the same values. Only at low loads do SPFF and NCR get a slight improvement with respect to the remaining algorithm. In the NCR, the allocation of PA slots is random within the supercycle slot window, so that in 

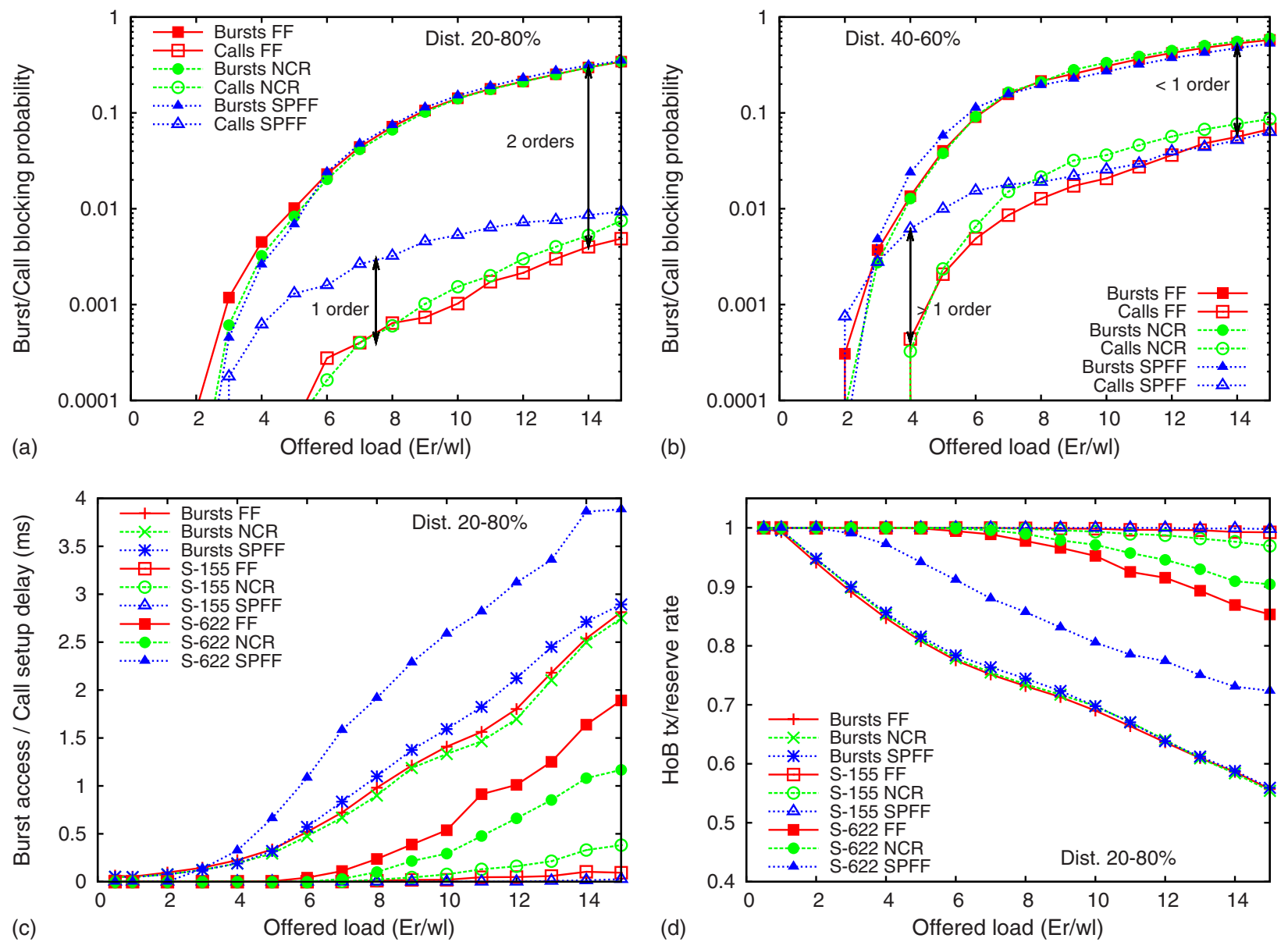

Fig. 14. (Color online) Dual PA/QA access modes. (a) Mean burst/call blocking probability (BP) on traffic distribution 20\%-80\%. (b) BP with traffic distribution $40 \%-60 \%$. (c) Mean access delay for connectionless bursts and mean connection setup delay on traffic distribution $20 \%-80 \%$. (d) HoB connectionless burst transmission and HoB connection setup rates on traffic distribution $20 \%-80 \%$.

between them connectionless bursts can be transmitted with lower waiting delay, thus decreasing the blocking probability. Regarding the call blocking probability (CBP) of the TDM services, this always remains below the $\mathrm{BBP}$ because the MAC protocol gives preference to the allocation of PA slots. Moreover, the mean CBP of SPFF is the greatest among the three algorithms, especially influenced by the call blocking of the $n$-slots connections due to the periodicity restrictions imposed by Eq. (3). Although the SPFF performance is not especially high, this type of algorithm provides extra functionalities for services that require restricted jitter and delay variations.

Furthermore, comparing both figures, we can see that the greater the connection-oriented offered traffic, the higher the BBP for the connectionless burst traffic and the higher the CBP for the TDM services. For instance, increasing the TDM load from $20 \%$ to $40 \%$ reduces the blocking probability difference between connectionless and connection-oriented calls to less than 1 order at high loads. This is due to the considerable increase of the CBP (by 1 order of magnitude). As a result, doubling the TDM traffic load in- creases both $\mathrm{BBP}$ and $\mathrm{CBP}$; hence a decrease of the carried traffic load is also expected.

In Fig. 14(c) the mean access delay for connectionless bursts and the mean connection setup delay for the connection-oriented services in the first traffic distribution scenario $(20 \%-80 \%)$ is shown. In general, connectionless bursts see an increase in their access delay due to the decrease of channel capacity left by the allocation of TDM services. Although the results are very similar using the three scheduling algorithms, a slightly shorter delay in the NCR can be observed. The connectionless burst access delay in the SPFF is slightly greater than the other two algorithms because in this case a greater number of connections are required by the core nodes in the lighttree; hence less network capacity is left to other upstream nodes between the $\mathrm{HoB}$ and the origin core nodes. With respect to the connection-oriented services, as expected, S-622 connections have a higher average call setup delay because a greater number of connections are initiated by the core nodes due to crankback. In such a case, the more restrictive the scheduling constraints, the longer the setup delay. For 
instance, because the SPFF algorithm constraints are the most restrictive, the setup delay for the $n$-slot connections is the largest. Unlike SPFF, the NCR algorithm allows the bandwidth connection to be set up randomly using the void slots on the slot window; hence it is less restrictive and therefore easier for the $n$-slot connections to be allocated.

Figure $14(d)$ shows the HoB burst transmission rate for the connectionless services and the connection setup rate when the origin node of the connectionoriented services is the HoB of the light-tree. This is an interesting performance parameter because it explains many of the results from previous paragraphs. In general, the higher the offered load to the network, the lower the $\mathrm{HoB}$ connectionless burst transmission rate. Besides, we can also see that the reservation rate of S-622 connections using the SPFF algorithm is the lowest in comparison with the other two algorithms, which corroborates the performance of the mean call setup delay seen in Fig. 14(c).

Finally, Fig. 15 shows in more detail the CBP distributed by algorithm and connection type, but now as a function of the mean network load. The CBP of S-622 connections using the SPFF gets the worst performance. This is because, even when enhancing the scheduling algorithm with slot sliding, the fact that the scheduling of slots must be done in accordance with Eq. (3) considerably reduces the slot allocation search space for $n$-slot connections. On the contrary, the S-155 connections that only require 1 slot can easily be allocated, and in fact, the blocking probability of S-155 turns out to be null for the whole load range. Moreover, the allocation of 1-slot connections decrements even more the search space for the $n$-slot connections, hence diminishing the setup successfulness ratio of these second connections. Regarding the other two scheduling algorithms, the CBP of S-622 connections using $\mathrm{FF}$ is higher than NCR because the slot allocation is continuous in the FF service type, whereas

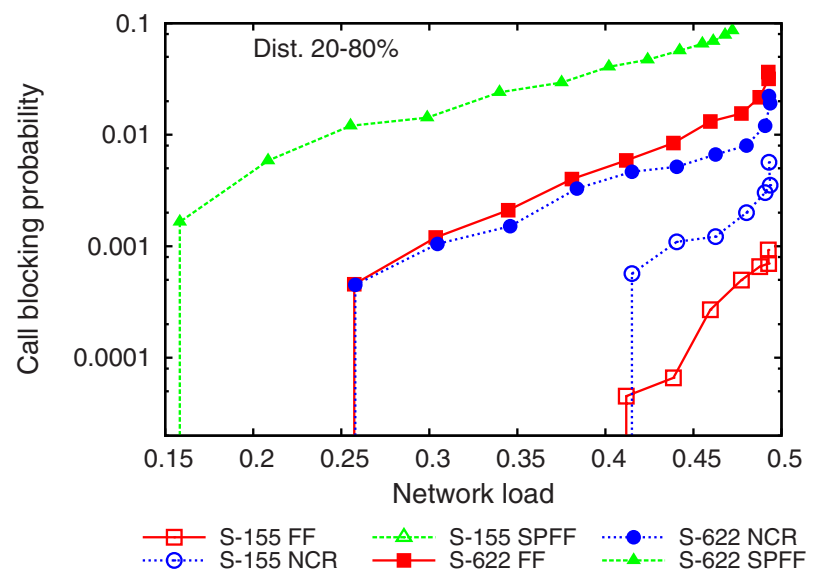

Fig. 15. (Color online) BP for different connection types on traffic distribution $20 \%-80 \%$.
NCR takes advantage of randomly scheduling the connections among the void slots so that a greater number of options are available given the same slot window size. However, S-155 connections get a slightly lower $\mathrm{CBP}$ using the $\mathrm{FF}$, merely due to the fact that S-622 connections get a higher CBP; hence more free space is available to allocate the remaining 1-slot connections.

Each algorithm delivers its optimum performance under specific traffic conditions. For this reason, introducing a scheduling machine able to make dynamic decisions and autonomously select the optimum algorithm may easily improve the overall PA slot reservation performance. The selection of the algorithm would need to take into account the type of service and the network status, e.g., resource utilization, to make sure that the slot reservations can be efficiently accommodated in the spare channel capacity. Moreover, in order not to disrupt the service of live connections (e.g., already set up), the algorithm optimization would need to be processed only for new connection requests.

\section{PA/QA Access Mode Transmission Decision}

In view of the results from previous analysis, in this subsection, a service and access mode assignment is proposed using the two DAOBS access schemes (PA and QA) and the QoS differentiation within the QA access mode itself. First of all, it is of interest to evaluate the burst blocking performance under similar scenarios. Figure 16 gives the BBP as a function of the mean offered load for the connectionless bursts $(80 \%$ of the load) when these are transmitted together with other connection-oriented TDM services $(20 \%$ of the load). Likewise, the mean BBP of the connectionless burst with only QA access supporting multiple QoS with the first traffic distribution from Table I is also

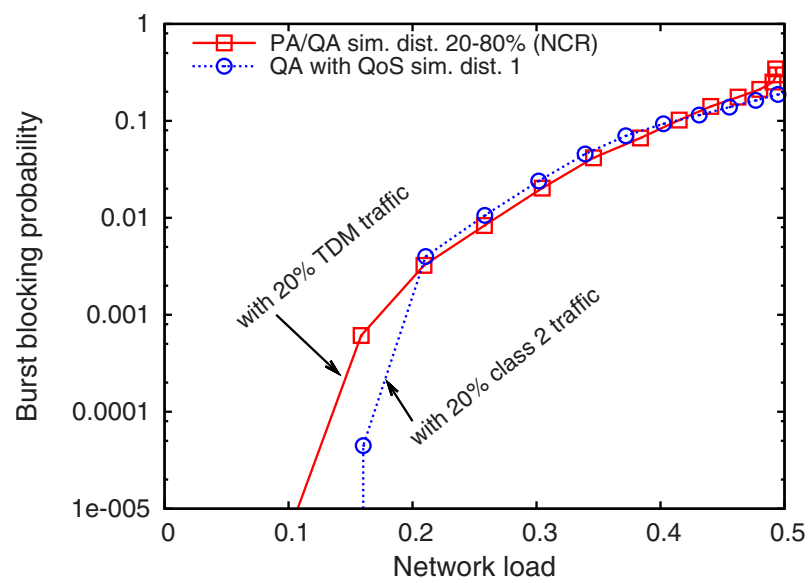

Fig. 16. (Color online) BBP comparison between standalone QA access with QoS and PA/QA dual access modes. 
represented. We have taken these two plots because in both cases the highest-priority traffic (connectionoriented traffic with PA access in the first case, and class 2 traffic in the second case) represents $20 \%$ of the offered load. As we can see, at high loads, the two plots lay within the same value range, with very slight differences between them. Therefore, it is expected that the class differentiation between the two lowest-priority traffics in the QA access mode will perform similarly even in the case that the channel is also shared with connection-oriented traffic using PA access, as long as the load of this traffic class is similar to class 2 traffic.

Turning to the performance of the highest-priority traffic in the circumstances mentioned in Fig. 16, the decision making of which traffic should be assigned as class 2 connectionless or as connection-oriented, depends specifically on the type of traffic and service required. In Subsection IV.B, the QoS differentiation provided by the QA access mode has been analyzed concluding that for both traffic distributions class 2 is lossless for the whole load range. On the contrary, from Subsection IV.C the connection-oriented traffic, which is given preference using the PA access mode, gets a nonnull CBP starting from medium offered loads but is still lower than the blocking probability of connectionless bursts. In view of this, class 2 QA traffic is envisioned for very high-priority connectionless traffic, such as control traffic without explicit jitter control guarantees, whereas PA access mode can be more suitable for services demanding a guaranteed bandwidth for a long period (in comparison with the burst size) that under some circumstances may also demand jitter and delay guarantees. As a concluding remark, Table III resumes the usage of each class of service provided by the multiservice DAOBS architecture.

TABLE III

DAOBS Classes OF SERVICE ${ }^{a}$

\begin{tabular}{|c|c|c|c|c|}
\hline Mode & $\mathrm{CoS}$ & Type & Use & Application \\
\hline \multirow[t]{3}{*}{ QA } & $\begin{array}{c}\text { Class } \\
0\end{array}$ & CL & $\begin{array}{l}\text { Best-effort traffic } \\
\text { without loss } \\
\text { guarantees or delay }\end{array}$ & $\begin{array}{l}\text { Transactional } \\
\text { data }\end{array}$ \\
\hline & $\begin{array}{c}\text { Class } \\
1\end{array}$ & CL & $\begin{array}{l}\text { Priority traffic that } \\
\text { tolerates some losses } \\
\text { without guaranteed } \\
\text { delay }\end{array}$ & $\begin{array}{l}\text { Interactive } \\
\text { audio/video }\end{array}$ \\
\hline & $\begin{array}{c}\text { Class } \\
2\end{array}$ & CL & $\begin{array}{l}\text { Very high-priority } \\
\text { traffic that does not } \\
\text { tolerate losses at all }\end{array}$ & $\begin{array}{l}\text { Interactive } \\
\text { data, } \\
\text { control }\end{array}$ \\
\hline $\mathrm{PA}$ & $\begin{array}{c}\text { Class } \\
3\end{array}$ & $\mathrm{CO}$ & $\begin{array}{l}\text { Long-life TDM traffic } \\
\text { with guaranteed } \\
\text { bandwidth and delay }\end{array}$ & $\begin{array}{l}\text { IPTV, cloud } \\
\text { computing, } \\
\text { grid }\end{array}$ \\
\hline
\end{tabular}

${ }^{a} \mathrm{CL}$, connectionless; $\mathrm{CO}$, connection-oriented.

\section{CONCLUSION}

This paper has introduced and extensively analyzed a novel multiservice MAC protocol for OBS mesh networks. An advantage of this protocol is its integrated design that permits it to potentially serve a broad range of applications with diverse QoS requirements. The MAC provides two main access methods: queue arbitrated for connectionless bursts and prearbitrated for TDM connection-oriented services. On the one hand, the queue-arbitrated access is based on a counting and monitoring process of burst and request control packets traveling in opposite control channel directions and a distributed preemption-based scheme in a multiqueue access priority system. On the other hand, the prearbitrated mode is based on the prereservation of slots supported by an upper service layer module. Apart from its simplicity and low implementation complexity, the protocol also offers great performance regarding the relative differentiation of QoS among different burst traffic classes in the QA access mode for both QoS parameters, burst blocking probability, and end-to-end delay. Results evaluated through simulations show that the highest-priority bursts are guaranteed zero losses and very low access latencies in the QA access mode. Even for the intermediate traffic class, the protocol can guarantee an acceptable BBP for a diverse number of applications. Regarding the PA access mode, results show that doubling the offered TDM traffic load increases the CBP by more than 1 order, slightly affecting the blocking of connectionless bursts. Moreover, three different slot scheduling algorithms for allocating TDM connections along with connectionless bursts have also been evaluated, providing diverse results depending on the requested bandwidth. The overall results demonstrate the suitability of the proposed architecture for future integrated multiservice optical networks.

\section{ACKNOWLEDGMENTS}

This work has been supported by the Government of Spain through project TEC2009-13901-C02-01, the EC-FP7 Euro-NF, the i2CAT Foundation's project TARIFA, and by the Government of Catalonia and the European Social Fund through a predoctoral scholarship (FI) and a BE fellowship.

\section{REFERENCES}

[1] G. Zervas, Y. Qin, R. Nejabati, D. Simeonidou, F. Callegati, A. Campi, and W. Cerroni, "SIP-enabled optical burst switching architectures and protocols for application-aware optical networks," Comput. Netw., vol. 52, no. 10, pp. 2065-2076, July 2008.

[2] Y. Chen, C. Qiao, and X. Yu, "Optical burst switching: a new area in optical networking research," IEEE Network, vol. 18, no. 3, pp. 16-23, May 2004.

[3] J. Triay, J. Perelló, C. Cervelló-Pastor, and S. Spadaro, "On 
avoiding-minimizing burst collisions in optical burst-switched networks without wavelength conversion," Proc. of 11th Int. Conf. on Transparent Optical Networks (ICTON), Azores, Portugal, 2009, paper Mo.D3.4.

[4] IEEE Standards Board, "Distributed queue dual bus (DQDB) subnetwork of a metropolitan network-802.6," Dec. 1990.

[5] H. Overby, "Quality of service differentation: teletraffic analysis and network layer packet redundancy in optical packet switched networks," Ph.D. dissertation, Dept. of Telematics, Norwegian University of Science and Technology, Trondheim, Norway, 2005.

[6] M. Yoo, C. Qiao, and S. Dixit, "QoS performance of optical burst switching in IP over WDM networks," IEEE J. Sel. Areas Commun., vol. 18, no. 10, pp. 2062-2071, Oct. 2000.

[7] H. Overby, N. Stol, and M. Nord, "Evaluation of QoS differentiation mechanisms in asynchronous bufferless optical packetswitched networks," IEEE Commun. Mag., vol. 44, no. 8, pp. 52-57, Aug. 2006.

[8] Q. Zhang, V. M. Vokkarane, J. P. Jue, and B. Chen, "Absolute QoS differentation in optical burst-switched networks," IEEE J. Sel. Areas Commun., vol. 22, no. 9, pp. 1781-1795, 2004.

[9] X. Liu, C. Qiao, W. Wei, and T. Wang, "A universal signaling, switching and reservation framework for future optical networks," J. Lightwave Technol., vol. 27, no. 12, pp. 1806-1815, June 2009.

[10] J. A. Hernández, P. Reviriego, J. L. García-Dorado, V. López, D. Larrabeiti, and J. Aracil, "Performance evaluation and design of polymorphous OBS networks with guaranteed TDM services," J. Lightwave Technol., vol. 27, no. 13, pp. 2495-2505, July 2009.

[11] L. Xu, H. G. Perros, and G. N. Rouskas, "A simulation study of optical burst switching and access protocols for WDM ring networks," Comput. Netw., vol. 41, no. 2, pp. 143-160, Feb. 2003.

[12] W. P. Chen, W. F. Wang, and W. S. Hwang, "A novel and simple beforehand bandwidth reservation (BBR) MAC protocol for OBS metro ring networks," J. High Speed Networks, vol. 17, no. 1, pp. 59-72, Jan. 2008.

[13] H. T. Lin and W. R. Chang, "CORnet: an OBS metro ring network with QoS support and fairness control," Comput. Netw., vol. 52, no. 10, pp. 2045-2064, July 2008.

[14] L. M. Peng and Y. C. Kim, "Investigation of the design of MAC protocols for TT-TR-based WDM burst-switched ring networks," J. Opt. Commun. Netw., vol. 1, no. 2, pp. 25-34, July 2009.

[15] Z. Zhang, L. Liu, and Y. Yang, "Slotted optical burst switching (SOBS) networks," Comput. Commun., vol. 30, no. 18, pp. 3471-3479, Dec. 2007.

[16] M. Klinkowski, D. Careglio, and J. Solé-Pareta, "Comparison of conventional and offset time-emulated optical burst switching," Proc. 8th Int. Conf. on Transparent Optical Networks (ICTON), Nothingham, UK, 2006, pp. 47-50.

[17] A. Gumaste, N. Ghani, P. Bafna, A. Lodha, A. Agrawal, T. Das, J. Wang, and S.-Q. Zheng, "DynaSPOT: dynamic services provisioned optical transport test-bed-achieving multirate multiservice dynamic provisioning using strongly connected lighttrail (SLiT) technology," J. Lightwave Technol., vol. 26, no. 1, pp. 183-195, Jan. 2008.

[18] B. Mukherjee, Optical WDM Networks, 1st ed. Springer, 2006.

[19] A. Rostami and A. Wolisz, "Modelling and synthesis of traffic in optical burst-switched networks," J. Lightwave Technol., vol. 25, no. 10, pp. 2942-2952, Oct. 2007.

[20] IST IP Nobel Phase 2: Next Generation Optical Networks for Broadband European Leadership Phase 2, Project deliverable D1.1: "Architectural Vision of Network Evolution," Aug. 2006.

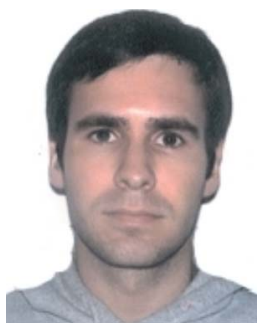

Joan Triay received a B.Eng. and a M.Eng. in telecommunications engineering and a M.Sc. in telematics engineering, in 2004, 2006, and 2007, respectively, all from Universitat Politècnica de Catalunya (UPC), Spain. In 2007, he was awarded with a 4-year predoctoral FI scholarship from the Government of Catalonia, and since then he has been a Ph.D. candidate in the Department of Telematics Engineering at UPC. He was a visiting fellow at the University of Essex (UK) from June 2009 to February 2010 thanks to a BE-DGR fellowship. His research interests include, but are not limited to, future optical network architectures and the provisioning of multiservice capabilities on high-speed networks.

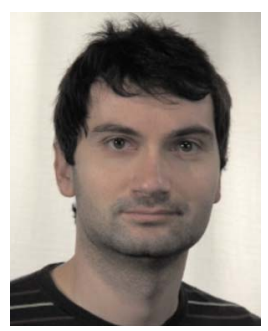

Georgios S. Zervas was awarded the M.Eng. degree in electronic and telecommunication systems engineering with distinction and the $\mathrm{Ph} . \mathrm{D}$. degree at the University of Essex (UK) in 2003 and 2008, respectively. He is a Senior Research Officer in the High Performance Networks Group at the University of Essex involved in current and past EC-funded projects MAINS, STRONGEST, GEANT3, BONE, Phosphorus MUFINS, and e-Photon/One+. He is author and co-author of over 50 papers in international journals and conferences. His research interests include high-speed optoelectronic router design, subwavelength networks (e.g., OBS), GMPLS networks, and grid networks. He is also involved in standardization activities in the Open Grid Forum (OGF) as an active member of the Grid High Performance Networking Research Group (GHPN-RG) and co-founder of the Network Service Interface Working Group (NSI-WG).

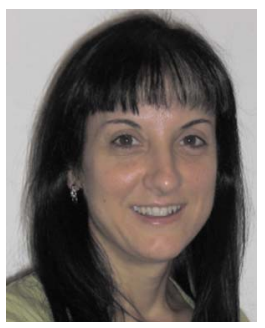

Cristina Cervelló-Pastor received her M.Sc. and Ph.D. in telecommunication engineering in 1989 and 1998, respectively, both from the Universitat Politècnica de Catalunya (UPC), Barcelona, Spain. She is currently an Associate Professor in the Department of Telematics Engineering at UPC, which she joined in 1989, and a leader of the optical networks research group within BAMPLA. Her research trajectory has been centered on the field of routing in highspeed networks and the development of new protocols and services in OBS/OPS, taking part in diverse national and European projects (FEDERICA, ATDMA, A@DAN, Euro-NGI, Euro-FGI, EURO-NF) and being responsible of various public and private funding $R \& D$ projects, some of them with the i2CAT Foundation. In parallel she has presented several patent proposals about OBS networks.

Dimitra Simeonidou is the Head of the High Performance Networks Group, which includes the Photonics Networks Laboratory and the newly established Network Media Laboratory, at the University of Essex, UK. She joined Essex in 1998 (previously with Alcatel Submarine Networks). While at Alcatel, she held the post of Senior Principle Engineer and contributed to the introduction of WDM in long-haul submarine links and pioneered the design and deployment of optical add-drop multiplexers. At Essex, she is leading a group of 30 researchers and Ph.D. students and she is involved in numerous national and international research projects. Her research is focusing in the fields of optical networks, grid and cloud computing, and the future Internet. She is author and coauthor of over 350 papers, 11 patents, and several standardization documents. 